\title{
ANÁLISE DOS PARÂMETROS DA EQUAÇÃO DE INFILTRAÇÃO DE KOSTIAKOV-LEWIS NA IRRIGAÇÃO POR SULCOS ${ }^{1}$
}

\author{
Luiz Carlos Almeida de Castro $^{2}$ \& Francisco de Souza ${ }^{3}$
}

\begin{abstract}
RESUMO
Este trabalho apresenta a análise das irrigações realizadas numa área da Fazenda Experimental do Perímetro Irrigado de Morada Nova, CE, de solo Aluvial eutrófico de textura franco-siltosa. Foram utilizados três sulcos-testes e realizadas quatro irrigações. Os dados de campo foram coletados e os resultados analisados, enfatizando-se o comportamento dos parâmetros de infiltração da equação de Kostiakov-Lewis e sua influência na infiltração acumulada; da análise, conclui-se que os valores de $a$ cresceram da primeira para a quarta irrigação, com exceção da segunda, sendo 0,1196, 0,0410, 0,2855 e 0,4529 , respectivamente, para as primeira, segunda, terceira e quarta irrigações. O parâmetro $k$ atingiu, para essas mesmas irrigações, os valores de $0,0156,0,0112,0,0066 \mathrm{e} 0,0024 \mathrm{~m}^{3} \mathrm{~min}^{-\mathrm{a}} \mathrm{m}^{-1}$. Com relação a $f_{0}$, infiltração básica, houve decréscimo até a terceira irrigação, alcançando uma estabilidade com os seguintes valores: $0,000516,0,000247,0,000179$ e $0,000170 \mathrm{~m}^{3} \mathrm{~min}^{-1} \mathrm{~m}^{-1}$.
\end{abstract}

Palavras-chave: infiltração, sulcos, Kostiakov-Lewis

\section{ANALYSIS OF THE PARAMETERS OF KOSTIAKOV-LEWIS INFILTRATION EQUATION FOR FURROW IRRIGATION}

\begin{abstract}
Field tests were conducted at the Experimental Farm of Morada Nova Irrigation District (Ceara, Brazil), using three $110 \mathrm{~m}$ long furrows laid on a $0.3 \%$ slope. The analysis consisted in verifying the variations of $a, k$ and $f_{0}$ parameters of Kostiakov-Lewis infiltration along the irrigation season. Results showed that $a$ values increased from 0.1196 in the first irrigation to 0.4529 in the last one, except for the second irrigation. Parameter $k$ decreased from $0.0156 \mathrm{~m}^{3} \mathrm{~min}^{-\mathrm{a}} \mathrm{m}^{-1}$ in the first irrigation, to $0.0112 \mathrm{~m}^{3} \mathrm{~min}^{-\mathrm{a}} \mathrm{m}^{-1}$ in the second, $0.0066 \mathrm{~m}^{3} \mathrm{~min}^{-\mathrm{a}} \mathrm{m}^{-1}$ in the third and $0.0024 \mathrm{~m}^{3} \mathrm{~min}^{-\mathrm{a}} \mathrm{m}^{-1}$ in the fourth; the values of $f_{o}$ varied in the following manner: $0.000516,0.000247,0.000179$ and $0.000170 \mathrm{~m}^{3} \mathrm{~min}^{-1}$.
\end{abstract}

Key words: infiltration, furrow, Kostiakov-Lewis

\section{INTRODUÇÃO}

Segundo Walker \& Skogerboe (1987) e Frizzone (1993) a infiltração é o fator mais importante na irrigação por superfície, pois controla a quantidade de água que entra no solo e, conseqüentemente, o avanço e a recessão; no entanto, prosseguem esses mesmos autores, a infiltração é, também, um dos mais difíceis parâmetros de serem determinados com precisão no campo. Pereira (1995) salienta que diversos modelos podem

\footnotetext{
${ }^{1}$ Este trabalho é parte integrante da dissertação de mestrado do primeiro autor, apresentada ao Departamento de Engenharia Agrícola da UFC, Fortaleza, CE

${ }^{2}$ Eng. Agr. M.Sc. Depto. de Eng. Agrícola/UFC, Fortaleza, CE, Fone: (085) 2889754

${ }^{3}$ Prof. Ph.D., Departamento de Engenharia Agrícola/UFC, Fortaleza, CE, E-mail:fsouza@ufc.br
} 
ser utilizados para descrever a infiltração da água e sua distribuição no perfil do solo.

Para Frizzone (1993) a infiltração é um processo complexo, dependente das propriedades físicas do solo, do seu conteúdo inicial de água, da forma de umedecimento e das variações de permeabilidade devido ao movimento da água na superfície e ao ar retido nos poros. Walker (1989) afirma que, em irrigação por superfície, a infiltração varia acentuadamente com a sequiência de irrigações. Segundo Frizzone (1993), isto é devido às variações que ocorrem na rugosidade e na geometria da superfície onde se verifica o escoamento.

Booher (1974), Walker \& Skogerboe (1987) e Bautista \& Wallander (1993) comentam que o adequado dimensionamento e o manejo dos sistemas de irrigação por superfície requerem o conhecimento detalhado das características de infiltração da água no solo, porém a complexidade do fenômeno dificulta a obtenção de uma função de infiltração representativa para todo o período de cultivo (Frizzone, 1993; Bautista \& Wallander, 1993).

A importância de se caracterizar a infiltração reside, especialmente, no fato desta influenciar no valor da vazão de entrada e no tempo de irrigação (Walker, 1989 e Childs et al., 1993).

Souza (1981) verificou que o maior efeito da constante $a$ está na forma da curva de infiltração acumulada e, conseqüentemente, na curva de avanço. $\mathrm{O}$ efeito das constantes $k$ e $a$ na infiltração acumulada é muito pequeno e suas variações não foram significativas na lâmina infiltrada acumulada. Variações na constante $k$, mantendo-se $a$ inalterado, provocaram variação no avanço e este se torna mais lento ou mais rápido se houver diminuição ou aumento de $k$.

Walker \& Skogerboe (1987) sugerem que o valor de $f_{0}$ é estatisticamente constante através da estação de cultivo e de sulco a sulco. As diferenças encontradas são devidas aos sulcos compactados pelas máquinas agrícolas e aos sulcos não compactados e entre sulcos com diferentes vazões ou entre sulcos com diferentes quantidades de restos vegetais.

Trabalhando com sulcos de precisão, Fangmeir \& Ramsey (1978) apud Andrade (1994) concluíram que a largura da base e o perímetro molhado podem ser usados para caracterizar a vazão de entrada em sulcos, mostrando que a taxa de infiltração foi correlacionada linearmente com o perímetro molhado.

O autor diz, também, que a infiltração é um processo complexo, dependente de inúmeras variáveis relacionadas às características físicas e morfológicas do solo, e apresenta dependência da umidade inicial no perfil e da condição da superfície que, por sua, vez é afetada pela geometria da seção de escoamento e pelo escoamento superficial, que determinam a condição física e a grandeza da área de infiltração. Strelkoff \& Souza (1984) sugerem que a altura da água em sulcos possui grande influência na infiltração.

Segundo Walker (1989) para a interpretação dos dados de campo é necessário que a infiltração seja representada por meio de um equação matemática. Diversas equações têm sido usadas, sendo que a mais comum é a de Kostiakov, em que a infiltração acumulada pode ser escrita por:

$$
\mathrm{Z}=\mathrm{kt}^{\mathrm{a}}
$$

em que:

$\mathrm{Z}$ - infiltração acumulada, $\mathrm{m}^{3} \mathrm{~m}^{-1}$

$\mathrm{t} \quad$ - tempo de oportunidade, minutos

k e $a$ - constantes empíricas.
Esta equação tem grande limitação, por não descrever bem a infiltração para longos períodos de tempo. A taxa de infiltração (I) obtida com base na equação 1 é:

$$
\frac{\partial \mathrm{Z}}{\partial \mathrm{t}}=\mathrm{I}=\mathrm{akt}^{\mathrm{a}-1}
$$

Para solucionar esse tipo de problema, foi desenvolvida uma equação designada de Kostiakov-Lewis modificada, à qual foi acrescentado um termo que considera a velocidade de infiltração básica da água no solo. Assim, a equação 1 pode ser escrita como:

$$
\mathrm{Z}=\mathrm{kt}^{a}+f_{0} \mathrm{t}
$$

em que $f_{0}$ é a velocidade de infiltração básica da água no solo em $\mathrm{m}^{3} \mathrm{~m}^{-1} \mathrm{~min}^{-1}$. A Equação 3 reduz-se à Equação 1 se $f_{0}$ se aproxima de zero ou se o tempo de irrigação é curto, comparado ao tempo requerido para que a infiltração alcance a velocidade de infiltração básica; no entanto, a Equação 3 aproxima-se melhor da condição real de infiltração em muitos tipos de solo.

A taxa de infiltração da água no solo obtida a partir da Equação 3 é:

$$
\frac{\partial \mathrm{Z}}{\partial \mathrm{t}}=\mathrm{I}=\mathrm{akt}^{\mathrm{a}-1}+f_{0}
$$

O objetivo do presente trabalho constitui-se em apresentar os aspectos relacionados à avaliação do comportamento dos parâmetros da equação de Kostiakov-Lewis e sua influência na infiltração acumulada, ao longo de quatro irrigações.

\section{MATERIAL E MÉTODOS}

O estudo foi realizado na Fazenda Experimental do Perímetro Irrigado de Morada Nova, distante $160 \mathrm{~km}$ de Fortaleza, CE. O solo da área experimental é do tipo Aluvial eutrófico, de textura franco-siltosa, com densidade aparente de $1,4 \mathrm{~g} \mathrm{~cm}^{-3}$ e valores de capacidade de campo e ponto de murcha de $26,5 \%$ e $10,0 \%$, respectivamente. A freqüência de irrigação foi calculada para o consumo de $50 \%$ da umidade disponível do solo.

Para se determinar os parâmetros da equação de KostiakovLewis, utilizou-se a metodologia dos dois pontos, proposta por Elliot \& Walker (1982) por ser mais simples e apresentar resultados compatíveis com os métodos tradicionais. Deste modo, os parâmetros $a, k$ e $f_{0}$ da Eq. 3 foram calculados de acordo com as seguintes expressões:

$$
\begin{gathered}
\mathrm{a}=\frac{\ln \left(\mathrm{V}_{\mathrm{L}} / \mathrm{V}_{0,5 \mathrm{~L}}\right)}{\ln \left(\mathrm{t}_{\mathrm{L}} / \mathrm{T}_{0,5 \mathrm{~L}}\right)} \\
\mathrm{k}=\frac{\mathrm{V}_{\mathrm{L}}}{\mathrm{S}_{\mathrm{Z}} \cdot \mathrm{t}_{\mathrm{L}}^{\mathrm{a}}}
\end{gathered}
$$

sendo:

$$
V_{L}=\frac{Q_{0} t_{L}}{L}-S_{y} A_{o}-\frac{f_{0} t_{L}}{(1+r)}
$$

$\mathrm{e}$

$$
\mathrm{V}_{0,5 \mathrm{~L}}=\frac{2 \mathrm{Q}_{0} \mathrm{t}_{0,5 \mathrm{~L}}}{\mathrm{~L}}-\mathrm{S}_{\mathrm{y}} \mathrm{A}_{0}-\frac{\mathrm{f}_{0} \mathrm{t}_{0,5 \mathrm{~L}}}{(1+\mathrm{r})}
$$


sendo que $S_{z}$ = fator de forma subsuperficial, obtido pela Eq. 9; $\mathrm{t}_{\mathrm{L}}=$ tempo de avanço correspondente à extremidade final do sulco e $\mathrm{t}_{0.5 \mathrm{~L}}$ é o avanço a $L / 2, \mathrm{Q}_{\mathrm{o}}=$ vazão por sulco $\left(\mathrm{m}^{3} \mathrm{~min}^{-1}\right), L$ é o comprimento do sulco (m); $S_{y}=$ fator de forma de perfil superficial; $A_{o}=$ área da seção transversal na entrada do sulco e $r=$ expoente da equação de avanço, a seguir.

$$
\mathrm{x}=\mathrm{p} \cdot \mathrm{t}_{\mathrm{x}}^{\mathrm{r}}
$$

$S_{z}$ é definido como:

$$
\mathrm{S}_{\mathrm{z}}=\frac{\mathrm{a}+\mathrm{r}(1-\mathrm{a})+1}{(1+\mathrm{r})(1+\mathrm{a})}
$$

O valor de $f_{0}$ é obtido pela equação:

$$
\mathrm{f}_{\mathrm{o}}=\frac{\left(\mathrm{Q}_{\mathrm{o}}-\mathrm{Q}_{\mathrm{ro}}\right)}{\mathrm{L}}
$$

em que, $Q_{r o}=$ vazão de escoamento superficial $\left(\mathrm{m}^{3} \min ^{-1}\right)$, na extremidade final do sulco.

A área da seção molhada na entrada do sulco pode ser calculada usando-se a equação de Manning:

$$
\mathrm{Ao}=\left[\frac{\mathrm{Q}_{0} \cdot \mathrm{n}}{60 \mathrm{p}_{1} \mathrm{~S}_{0}^{0,5}}\right]^{1 / \mathrm{P}_{2}}
$$

sendo, $P_{1}$ e $P_{2}$ fatores de forma, e $S_{0}$ é a declividade do sulco, em $\mathrm{mm}^{-1}$.

Para os testes de campo utilizaram-se três sulcos-teste, cada um ladeado por outros dois, que serviram como bordadura; os sulcos tinham comprimento de $110 \mathrm{~m}$ com $1 \mathrm{~m}$ de espaçamento e declividade de $0,3 \%$. As vazões eram medidas em calhas Parshall de uma polegada, previamente calibradas, a primeira instalada no início do sulco, para medir as vazões de entrada, e a segunda no final do sulco, para medir as vazões de saída, com o objetivo de se construir os hidrogramas de entrada e saída, respectivamente. A água era conduzida ao local através de canais de terra situados à montante e, à jusante, havia um dreno parcelar para coletar a água perdida por escoamento superficial (runoff).

Realizaram-se quatro irrigações, com intervalo de oito dias entre uma e outra, de forma a se atingir a velocidade de infiltração básica da água no solo; para tanto, fazia-se o corte da água somente após um período considerado, em que a vazão permanecia constante na calha de saída.

A área foi semeada com caupi (Vigna unguiculata (L.) Walp) variedade EPACE-10 que apresenta ciclo de 65 a 75 dias. Observou-se que o desenvolvimento da cultura, ao longo do ciclo vegetativo, com o crescimento da área foliar, foi um fator que funcionou retardando o fluxo superficial, com o aumento da rugosidade hidráulica do sulco. Após a quarta irrigação, as medições foram prejudicadas em virtude da grande massa vegetal que se formou sobre o sulco, dificultando as medições de avanço e recessão. Deve-se considerar, também, que o desenvolvimento de ervas daninhas concorre para variação das condições do fluxo de avanço da água.

Deste modo, entre a segunda e a terceira irrigação houve a necessidade de eliminação das ervas daninhas que se desenvolveram na área; fez-se, então, uma erradicação dessas ervas, manualmente, o que alterou, mais uma vez, as condições de fluxo superficial, afetando a rugosidade hidráulica do sulco.

Para o estabelecimento das curvas de avanço e recessão, instalaram-se doze estacas, distanciadas $10 \mathrm{~m}$ uma da outra e, também, uma estaca intermediária aos $55 \mathrm{~m}$ (metade do campo) sendo que nesta só se registrava o tempo de avanço.

Para se descrever a geometria do sulco, utilizou-se um perfilômetro, durante a fase de avanço, onde se fazia uma medida junto à estaca 3 e outra junto à estaca 4 . Plotou-se em papel milimetrado a seção do sulco obtida no campo, a qual se dividiu em 10 a 15 intervalos iguais; desta obteve-se a largura da superfície da água e a metade da lâmina de água. Observou-se que os sulcos, que inicialmente tinham forma triangular, modificavam-se durante a fase de avanço, para uma forma parabólica.

\section{RESULTADOS E DISCUSSÃO}

Na Tabela 1 observa-se a variação dos parâmetros da equação de Kostiakov-Lewis $\left(a, k\right.$ e $\left.f_{0}\right)$ para as quatro irrigações realizadas. Nesta tabela verifica-se, também, que o valor de $a$ aumentou durante a estação de cultivo. Uma exceção das demais irrigações observa-se na segunda, onde o valor deste parâmetro se apresenta mais baixo e, com relação ao parâmetro $k$, pode-se observar que este sofreu decréscimo.

Conforme estabelece a literatura, a infiltração é um processo complexo, dependente das propriedades físicas do solo, do seu conteúdo de água, da forma de umedecimento, das variações da rugosidade e da geometria do sulco. Esses fatores devem ser considerados amplamente na análise dos resultados.

Tabela 1. Parâmetros da equação de infiltração de KostiakovLewis, ao longo das 4 irrigações

\begin{tabular}{cccc}
\hline \multirow{2}{*}{ Irrigações } & \multicolumn{3}{c}{ Parâmetros } \\
\cline { 2 - 4 } & $\mathrm{a}$ & $\mathrm{k}\left(\mathrm{m}^{3} \mathrm{~min}^{-\mathrm{a}} \mathrm{m}^{-1}\right)$ & $\mathrm{f}_{\mathrm{o}}\left(\mathrm{m}^{3} \mathrm{~min}^{-1} \mathrm{~m}^{-1}\right)$ \\
\hline 1 & 0,1196 & 0,0156 & 0,000516 \\
2 & 0,0410 & 0,0112 & 0,000247 \\
3 & 0,2855 & 0,0066 & 0,000179 \\
4 & 0,4529 & 0,0024 & 0,000170 \\
\hline
\end{tabular}

Considerando-se que o solo da área experimental - Aluvial eutrófico - apresenta grande variabilidade espacial pelo próprio processo de formação de um solo aluvial, com capacidade de retenção de $115,5 \mathrm{~mm} \mathrm{~m}^{-1}$, para um consumo de $50 \%$ da água disponível, procurou-se investigar o comportamento da infiltração durante as quatro irrigações, ao longo do ciclo da cultura, com base nos demais parâmetros. A forma de umedecimento do solo obedecia a uma frequiência de irrigação a cada 8 dias. A geometria do sulco, por sua vez, era variável, uma vez que se observaram mudanças na forma do sulco, já na fase de avanço, por efeito erosivo. Ao longo do ciclo da cultura, durante as quatro irrigações, a geometria foi alterada em função, também, do desenvolvimento vegetativo da cultura; este, por sua vez, tinha influência no incremento da rugosidade hidráulica do sulco, pois a folhagem se constituía, a medida em que as plantas cresciam, em fator físico impeditivo ao livre fluxo do avanço da água e isto retardava a frente de avanço e tinha consequiências sobre os parâmetros da equação de Kostiakov- 
Lewis, ao longo do ciclo e, conseqüentemente, sobre a performance do sistema.

Outro aspecto a ser considerado na análise foi o próprio processo de cultivo, com a eliminação das ervas daninhas que se desenvolveram na área. $\mathrm{O}$ trabalho realizado a tração animal com acabamento manual, deve ter provocado alterações nas condições de rugosidade hidráulica e, principalmente, no selamento superficial, que é um fator importante na redução de $f_{0}$.

Como a vazão é influenciada pela maioria desses fatores, mesmo não tendo relação direta com a equação de infiltração, é que se tratou de analisar $a$, $k$ e $f_{0}$ com relação à vazão.

Os parâmetros $a$ e $k$ foram relacionados com a vazão aplicada, na tentativa de se investigar as razões da sua variação durante as quatro irrigações. Através de regressão linear e comparando-se $a$ e $Q_{0}$ verificou-se que este parâmetro não é influenciado pela vazão, já que o coeficiente de determinação entre $a$ e a vazão aplicada é de 0,0256 (Tabela 2).

Observando-se a Tabela 2, pode-se verificar que a constante $k$ também não apresenta boa correlação linear com a vazão, indicando que este parâmetro não é influenciado diretamente pela vazão; o coeficiente de determinação encontrado neste caso foi de 0,3652 .

Tabela 2. Análise da influência da vazão aplicada sobre o parâmetro a da equação de Kostiakov-Lewis

\begin{tabular}{cccc}
\hline Irrigação & $\begin{array}{c}\text { Vazão aplicada } \\
\left(\mathrm{m}^{3} \min ^{-1}\right)\end{array}$ & $\mathrm{a}$ & $\begin{array}{c}\mathrm{k} \\
\left(\mathrm{m}^{3} \mathrm{~min}^{-\mathrm{a}} \mathrm{m}^{-1}\right)\end{array}$ \\
\hline 1 & 0,0738 & 0,1196 & 0,0156 \\
2 & 0,0449 & 0,0410 & 0,0112 \\
3 & 0,0565 & 0,2855 & 0,0066 \\
4 & 0,0503 & 0,4529 & 0,0024 \\
$\mathrm{R}^{2}$ & & 0,0256 & 0,3652 \\
\hline
\end{tabular}

Observando-se os dados apresentados na Tabela 1, verifica-se que o valor de $f_{0}$ (velocidade de infiltração básica) apresenta tendência de diminuir, com as irrigações. Isto se deve ao processo de selamento superficial que ocorreu, fato observado pelo arraste de material durante o avanço da água; no entanto, este processo ocorreu com maior intensidade nas primeiras irrigações.

Como se observa, o valor de $f_{0}$ não sofreu variação muito grande entre a terceira e a quarta irrigação, atingindo estabilidade entre elas.

Fazendo-se uma análise da influência da vazão e do volume aplicados sobre $f_{0}$, elaborou-se a Tabela 3. Observa-se que o coeficiente de determinação entre $Q_{0}$ e $f_{0}$ é de 0,7 ; já na relação entre o volume aplicado e a velocidade de infiltração básica, este coeficiente é de 0,98 , sugerindo que este parâmetro pode ser influenciado pela vazão aplicada e pelo tempo de corte. Tal comportamento já seria esperado, tendo em vista que a frente de umedecimento se aprofunda a medida em que aumenta o volume aplicado e, conseqüentemente, a velocidade de infiltração tende a aproximar-se de $f_{0}$.

Uma das razões que justificam a redução de $f_{0}$ com as irrigações, deve-se ao processo de carreamento das partículas mais finas de um local para outro, preenchendo os espaços porosos e diminuindo a infiltração do solo.
Tabela 3. Análise da influência da vazão aplicada e do volume sobre a velocidade de infiltração básica

\begin{tabular}{cccccc}
\hline Irrigação & $\begin{array}{c}\text { Vazão aplicada } \\
\left(\mathrm{m}^{3} \mathrm{~min}^{-1}\right)\end{array}$ & $\begin{array}{c}\text { Volume aplicado } \\
\left(\mathrm{m}^{3}\right)\end{array}$ & $\begin{array}{c}\text { fo } \\
\left(\mathrm{m}^{3} \mathrm{~min}^{-1} \mathrm{~m}^{-1}\right)\end{array}$ & $\begin{array}{c}\mathrm{R}^{2} \\
(\mathrm{Q} o . f o)\end{array}$ & $\begin{array}{c}\mathrm{R}^{2} \\
(\text { Va.fo })\end{array}$ \\
\hline 1 & 0,0738 & 9,4 & $5,16 \times 10^{-4}$ & & \\
2 & 0,0449 & 5,6 & $2,47 \times 10^{-4}$ & & \\
3 & 0,0565 & 5,3 & $1,79 \times 10^{-4}$ & & \\
4 & 0,0503 & 5,1 & $1,70 \times 10^{-4}$ & 0,7094 & 0,98 \\
\hline
\end{tabular}

Apesar de Walker \& Skogerboe (1987) terem sugerido que $f_{0}$ é estatisticamente constante através da estação de cultivo, isto não foi constatado neste trabalho, cujos valores de $\mathrm{f}_{0}$ sofreram redução ao longo das quatro irrigações.

\section{Variação percentual das constantes $a, k$ e $f_{0}$ em relação à infiltração acumulada}

A análise aqui apresentada tem o objetivo exclusivo de mostrar as variações percentuais das constantes $a, k$ e $f_{0}$ em relação à infiltração acumulada, sem pretensão de se apresentar contribuição científica expressiva mas, apenas, uma análise do comportamento de uma equação matemática, que representa o fenômeno mais importante na irrigação por superfície e, também, um dos mais difíceis de serem determinados com precisão no campo, de acordo com a literatura.

Portanto, para se verificar a influência da constante $a$ sobre a curva de infiltração acumulada, elaborou-se a Figura 1, na qual se apresentam as variações de $a$, onde foram utilizados os valores obtidos para os eventos 1, 2 e 3 . Para um tempo simulado de 360 min promoveu-se um decréscimo de $66 \%$ de $a_{1}$ para $a_{2} \mathrm{o}$ que representou uma diminuição de $5 \%$ na infiltração acumulada e o incremento de $138 \%$ de $a_{1}$ para $a_{3}$ causou uma elevação de $24 \%$ na mesma.

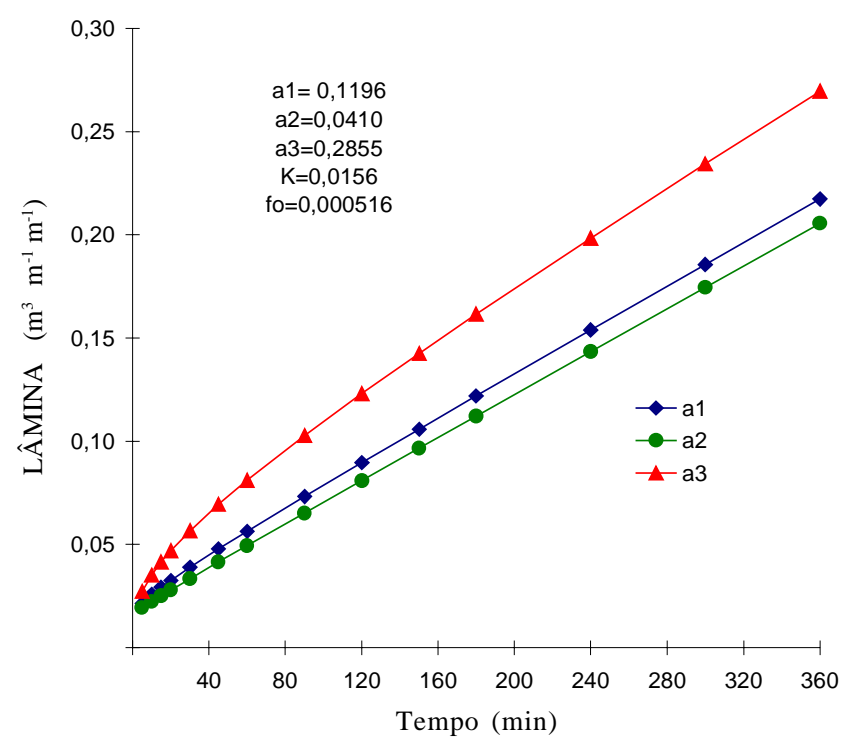

Figura 1. Efeito da variação do expoente $\boldsymbol{a}$ da equação de Kostiakov-Lewis sobre a infiltração acumulada

Em relação à constante $k$, utilizaram-se também os valores obtidos nas primeira, segunda e terceira irrigações, mantendo-se constantes os valores de $a$ e $f_{0}$. Observou-se que, variando $k_{1}$ para $k_{2}$ de $28 \%$, este representa redução de apenas $4 \%$ na infiltração acumulada e, diminuindo-se o valor de $k_{2}$ para $k_{3}$ em $41 \%$, o decréscimo gerado na infiltração 
acumulada foi de 4,5\% (Fig. 2). Verificou-se, também, a influência direta de $f_{0}$ (velocidade de infiltração básica) sobre a infiltração acumulada. Mantendo-se $a$ e $k$ constantes, utilizaram-se os três valores de $f_{0}$, para as primeira, segunda e terceira irrigações. Os valores de $a$ e $k$ utilizados foram os obtidos para a primeira irrigação; assim, do primeiro evento para o segundo, o decréscimo de $f_{0}$ foi de $52 \%$ e do segundo para o terceiro foi de $28 \%$; o primeiro decréscimo gerou uma diminuição de $45 \%$ na infiltração e o segundo redução de 20\% (Fig. 3).

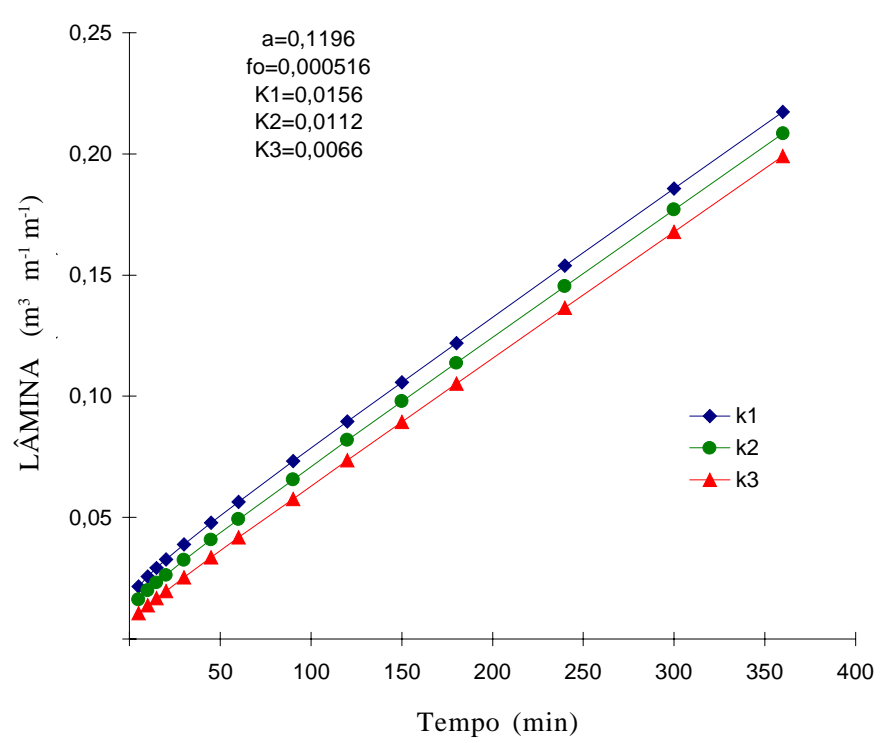

Figura 2. Efeito da variação da constante $k$ da equação de Kostiakov-Lewis sobre infiltração acumulada

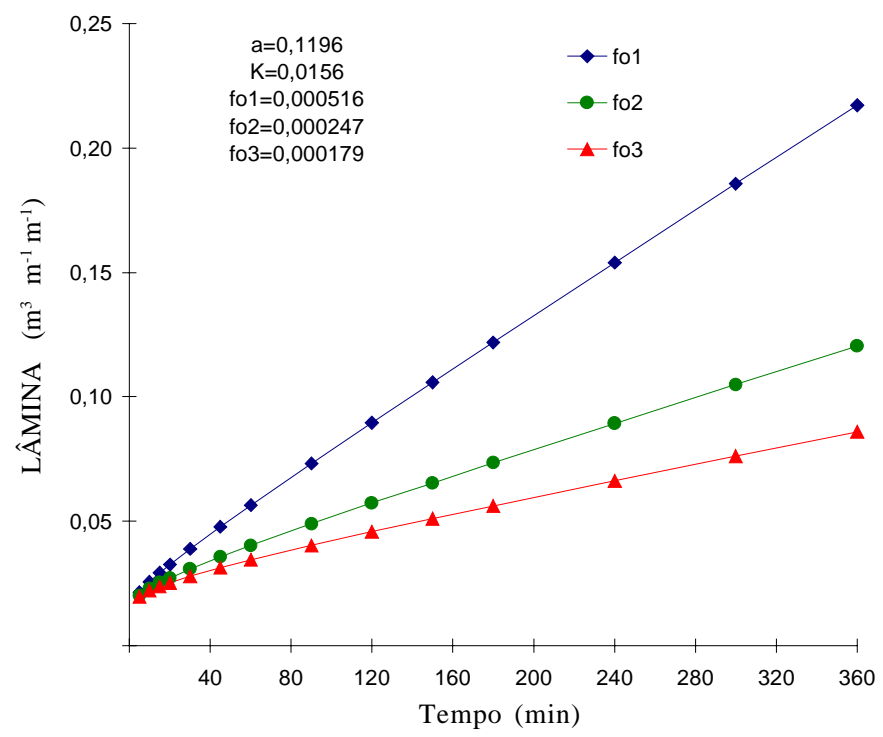

Figura 3. Efeito da variação da constante $f_{0}$ da equação de

Kostiakov-Lewis sobre a infiltração acumulada

Diferentemente do comportamento dos parâmetros $a$ e $k$ de que variações elevadas em seus valores originais geravam pequenas variações na infiltração acumulada, alterações na velocidade de infiltração básica provocaram variações da mesma magnitude na infiltração acumulada.

\section{Comportamento da infiltração ao longo do ciclo}

A Figura 4 apresenta a variação da taxa de infiltração da água no solo (I) para as quatro irrigações aplicadas ao longo do ciclo da cultura. Observa-se que a velocidade de infiltração foi reduzida da primeira para a segunda irrigação em torno de 53\%, a partir de um tempo simulado de $60 \mathrm{~min}$. Entre as segunda, terceira e quarta irrigações nota-se, para esse mesmo tempo simulado, uma pequena variação entre os valores de $I$, em que as diferenças são de $7 \%$ entre a segunda e a terceira irrigação e de $3 \%$ entre a terceira e a quarta, indicando uma estabilização a partir da segunda irrigação; no entanto, observa-se, desde o tempo de 5 min até 90 min, que os valores nas terceira e quarta irrigações são superiores ao da segunda irrigação, provavelmente em virtude do cultivo realizado, que revolveu as camadas superiores do solo, deixando as mais inferiores intactas.

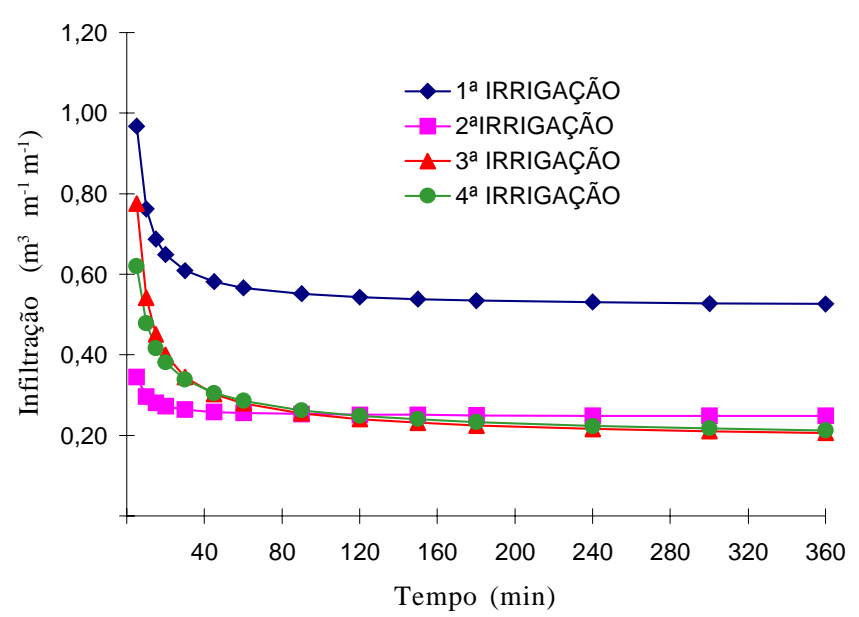

Figura 4. Velocidade de infiltração ao longo das quatro irrigações

\section{CONCLUSÕES}

O valor de $a$ cresceu ao longo das irrigações, com exceção do encontrado na segunda irrigação; $k$ decresceu para estas mesmas irrigações e o valor de $f_{o}$ apresentou o mesmo comportamento até a terceira irrigação, quando alcançou estabilização. Certamente, a variação da rugosidade hidráulica e do selamento superficial, em função do crescimento da massa foliar da cultura e do uso da tração animal na eliminação das ervas daninhas, foram fatores fundamentais na variação dos parâmetros da equação de Kostiakov-Lewis de acordo com as condições predominantes na área experimental. Sem dúvida, em virtude da importância da infiltração para a irrigação por superfície e das dificuldades na sua determinação com maior acuracidade, maiores investigações devem continuar a ser desenvolvidas.

\section{REFERÊNCIAS BIBLIOGRÁFICAS}

ANDRADE, D.V. Modelo matemático para simulação da fase de avanço na irrigação por sulcos. São Paulo: ESALQ/USP, 1994. 135p. Tese Doutorado

BAUTISTA, E.; WALLANDER, W.W. Numerical calculations of infiltration in furrow irrigation simulation models. Journal of Irrigation and Drainage Engineering, New York, v.119, n. 2, p. 286-311, 1993. 
BOOHER, L.J. Surface irrigation. Rome: FAO, 1974, 160p. FAO, 95

CHILDS, J.L.; WALLANDER, W.W.; HOPMANS, J.W. Spatial and variation of furrow infiltration. Journal of Irrigation and Drainage Engineering. New York, v. 119, n.1, p. 74-90, 1993.

ELLIOT, R.; WALKER, W.R. Field evaluation of furrow infiltration and advance functions. Transactions of the ASAE, v.25, n.2, p. 396-400, 1982.

FRIZZONE, J.A. Irrigação por superfície. São Paulo: ESALQ/ USP, 1993. 183p.

PEREIRA, L.S. Surface irrigation system. In: PEREIRA, L.S. (ed.) Sustainability of water resources utilization in agriculture. 1995.
SOUZA, F. de. Nonlinear hydrodynamic model of furrow irrigation. Davis California: University of California, 1981. 172p. Thesis Ph.D.

STRELKOFF, T.; SOUZA, F. de. Modeling effect of depth on furrow infiltration. Journal of the Irrigation and Drainage Engineering, New York, v. 110, n. 4, p. 375-387, 1984.

WALKER, W.R. Guidelines for designing and evaluating surface irrigation systems, Rome: FAO, 1989, 138p. FAO. Irrigation and Drainage, N. 45.

WALKER, W.R.; SKOGERBOE, G.V. Surface irrigation: Theory and practice. New Jersey: Prentice-Hall, 1987. 386 . 